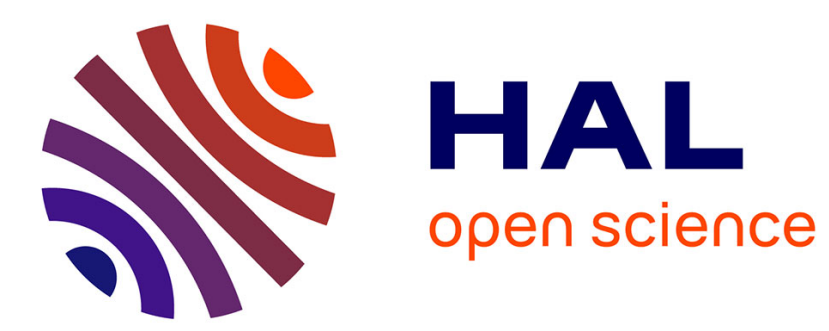

\title{
Climbing: A Unified Approach for Global Constraints on Hierarchical Segmentation
}

\author{
Bangalore Ravi Kiran, Jean Serra, Jean Cousty
}

\section{To cite this version:}

Bangalore Ravi Kiran, Jean Serra, Jean Cousty. Climbing: A Unified Approach for Global Constraints on Hierarchical Segmentation. Computer Vision - ECCV 2012. Workshops and Demonstrations, Oct 2012, Italy. pp.324-334. hal-00789392

\section{HAL Id: hal-00789392 \\ https://hal.science/hal-00789392}

Submitted on 18 Feb 2013

HAL is a multi-disciplinary open access archive for the deposit and dissemination of scientific research documents, whether they are published or not. The documents may come from teaching and research institutions in France or abroad, or from public or private research centers.
L'archive ouverte pluridisciplinaire HAL, est destinée au dépôt et à la diffusion de documents scientifiques de niveau recherche, publiés ou non, émanant des établissements d'enseignement et de recherche français ou étrangers, des laboratoires publics ou privés. 


\title{
Climbing: A Unified Approach for Global Constraints on Hierarchical Segmentation
}

\author{
Bangalore Ravi Kiran, Jean Serra, and Jean Cousty \\ Université Paris-Est, Laboratoire d'Informatique Gaspard-Monge, A3SI, ESIEE
}

\begin{abstract}
The paper deals with global constraints for hierarchical segmentations. The proposed framework associates, with an input image, a hierarchy of segmentations and an energy, and the subsequent optimization problem. It is the first paper that compiles the different global constraints and unifies them as Climbing energies. The transition from global optimization to local optimization is attained by the $h$-increasingness property, which allows to compare parent and child partition energies in hierarchies. The laws of composition of such energies are established and examples are given over the Berkeley Dataset for colour and texture segmentation.
\end{abstract}

\section{Motivation}

There have been multiple approaches to image segmentation by global constraint models. Some of them emphasize the use of seeds, e.g. the labels in graph-cuts, or the markers in watersheds. In addition, they view the space as a one scale structure. This line of thought is illustrated by the search for a maximum flow in a directed graph, or by the optimization of a conditional random field(CRF). At the opposite end, other segmentation approaches emphasize the scaling of the space by means of hierarchies, and attach less importance to labelling questions, in a first step at least.

The present paper is devoted to this second type of global constraints. A hierarchy, or pyramid, of image segmentations is classically understood as a series of progressive simplified versions of an initial image, which result in increasing partitions of the space. In the following, we do not aim to focus on the methods for obtaining pyramids of segmentation, and consider rather the whole hierarchies as a starting point 1 . Indeed, a multi-scale image description can rarely be considered as an end in itself. It often requires to be completed by some energy function that allows us to formalize optima, and to summarizes a hierarchy into some "optimal cut". Three questions arise then, namely:

1 The main techniques for hierarchical segmentation include functional minimizations of Mumford and Shah type, semi-groups of morphological filters, and progressive floodings on watersheds [1. In addition, the learning strategies for segmentation, as developed by [2], or by [3], among others, lead to very significant hierarchies. One can also quote [4] where Mumford and Shah functional is modified by shape descriptors.

A. Fusiello et al. (Eds.): ECCV 2012 Ws/Demos, Part III, LNCS 7585, pp. 324-334, 2012.

(C) Springer-Verlag Berlin Heidelberg 2012 
1. given a hierarchy $H$ of partitions and a an energy $\omega$ on the partial partitions, how to combine the classes of this hierarchy for obtaining a new partition that minimizes $\omega$, and which can be determined easily, in one ascending pass for example? In other words, how to characterize the convenient energies?

2. most of the segmentations involve several features (colour, shape, size, etc.), which give birth to several energies $\omega$. How to combine them?

3. when one energy $\omega$ depends on an integer $j$, i.e. $\omega=\omega^{j}$, how to generate a sequence of minimum partitions that increase with $j$, which therefore should form a minimum hierarchy?

These questions have been taken up by several authors, for many years, and by various methods. The most popular energies $\omega$ for hierarchical partitions derive from that of Mumford and Shah, in which a term of fidelity to the data is summed up with a term of boundary regularization. The optimization turns out to be a trade off between these two constraints. The method initiated by $\mathrm{Ph}$. Salembier and L. Garrido for generating thumbnails is of this type [5]. They interpret the best cut as the most accurate image simplification for a given compression rate. The approach has been extended to additive energies by L.Guigues et al [6]. It is always assumed, in all these studies, that the energy of any partial partition equals the sum of the energies of its classes, which considerably simplifies the combinatorial complexity, and answers the above two questions 1 and 3 .

However, one can wonder whether additivity is the very underlying cause of the nice properties, since P. Soille's constraint connectivity [7], though non linear, satisfies similar properties. It is also the case for F. Zanoguerra's lasso, which labels a foreground inside a given contour [8]. In these two cases, the addition is replaced by the supremum operation. Finally, one finds in literature a third type of energy, illustrated by Akçay and Aksoy, in [9] which holds on nodes only, and no longer on partial partitions. And again, it yields to best cuts, which are accessed in one pass.

Is there a common denominator to all these approaches, more comprehensive than just additivity? For solving problem 1 above, the alternative and simpler condition of $h$-increasingness (for hierarchical increasingness), proposed in [10] encompasses all above energy optimizations. But does it suffice for solving the two other points? This will be the matter of this paper, where the three above questions correspond to the next three sections, followed by a few examples.

Going back to the comparison between hierarchies, and CRF and graph mincuts methods, one can notice that:

1. The CRFs and min-cut max-flow formulation represent spatial interaction between pixels which is restricted in a unitary neighbourhood, and the increased complexity may not be always advantageous [1].

2. Hierarchical methods provide a lower combinatorial complexity while supplying intuitive segmentations. In addition, the construction of a hierarchy and of the ulterior energy $\omega$ may use independent pieces of information (e.g. in section 5, luminance based energy, versus chrominance and texture) . 


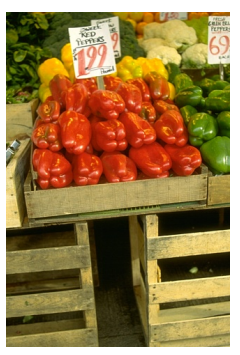

(a) Input Image

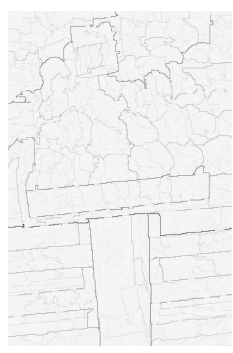

(b) Saliency

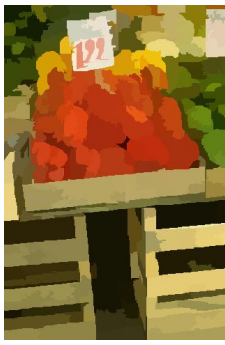

(c) level 3

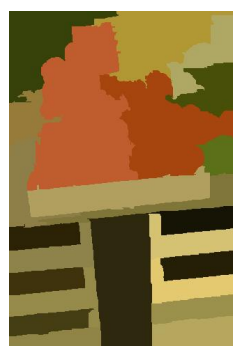

(d) level 6

Fig. 1. Saliency map and hierarchy of segmentations at few levels and their child-parent correspondences

3. Indirectly, the hierarchical clustering induces larger and larger neighbourhoods. This helps to differentiate textures at various scales, to progressively localize a significant detail, and to optimize image compression.

\subsection{Notation and Terms}

The space under study (euclidean $R^{n}$, digital $Z^{n}$, or otherwise) is denoted by $E$. A partition $\pi(S)$ associated with a set $S \in \mathcal{P}(E)$ is called partial partition of $E$ of support $S$ [12]. The family of all partial partitions of set $E$ is denoted by $\mathcal{D}(E)$, or simply by $\mathcal{D}$. A hierarchy $H$ is a finite chain of partitions $\pi_{i}$, i.e.

$$
H=\left\{\pi_{i}, 0 \leq i \leq n \mid i \leq k \leq n \Rightarrow \pi_{i} \leq \pi_{k}\right\},
$$

where $\pi_{n}$ is the partition $\{E\}$ of $E$ in a single class.

The partitions of a hierarchy may be represented by their classes, or by the saliency map of the edges [13, [1], as depicted in Figure 1, or again by a family tree where each node of bifurcation is a class $S$, as depicted in Figure 2. The classes of $\pi_{i-1}$ at level $i-1$ which are included in class $S_{i}$ are said to be the sons of $S_{i}$. Denote by $\mathcal{S}(H)$ the set of all classes $S$ of all partitions involved in $H$. Clearly, the descendants of each $S$ form in turn a hierarchy $H(S)$ of summit $S$, which is included in the complete hierarchy $H=H(E)$.

Cuts in a hierarchy Any partition $\pi$ of $E$ whose classes are taken in $\mathcal{S}$ defines a cut $\pi$ in a hierarchy $H$. The set of all cuts of $E$ is denoted by $\Pi(E)=\Pi$. Every "horizontal" section $\pi_{i}(H)$ at level $i$ is obviously a cut, but several levels can cooperate in a same cut, such as $\pi\left(S_{1}\right)$ and $\pi\left(S_{2}\right)$, drawn with thick dotted lines in Figure 2. Similarly, the partition $\pi\left(S_{1}\right) \sqcup \pi\left(S_{2}\right)$ generates a cut of $H(E)$. The symbol $\sqcup$ is used here for expressing that groups of classes are concatenated. Let $\Pi(S)$ be the family of all cuts of $H(S)$.

\section{Hierarchical Increasingness}

This section is a reminder of [10] when the $h$-increasingness was introduced first. 


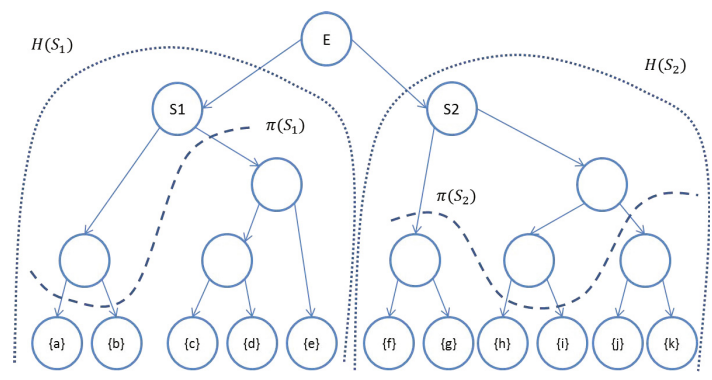

Fig. 2. The hierarchy: $S_{1}$ and $S_{2}$ are the nodes sons of $E$, and $H\left(S_{1}\right)$ and $H\left(S_{1}\right)$ are the associated sub-hierarchies. $\pi_{1}$ and $\pi_{2}$ are cuts of $H\left(S_{1}\right)$ and $H\left(S_{1}\right)$ respectively, and $\pi_{1} \sqcup \pi_{2}$ is a cut of $E$.

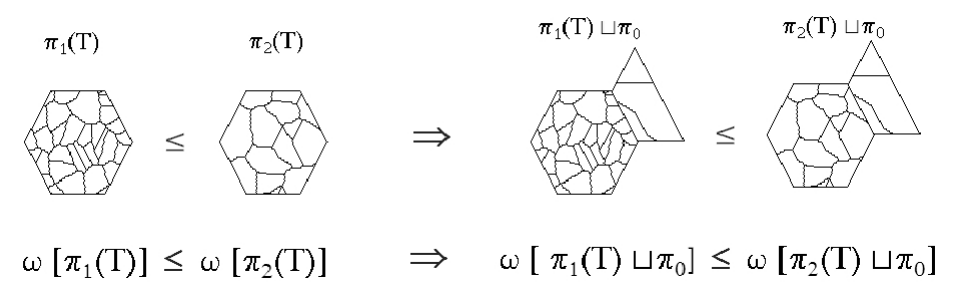

Fig. 3. Hierachical increasingness

Cuts of minimum energy and h-increasingness An energy $\omega: \mathcal{D}(E) \rightarrow \mathbb{R}^{+}$is a non negative numerical function over the family $\mathcal{D}(E)$ of all partial partitions of set $E$, and an optimal cut $\pi^{*} \in \Pi(E)$ of $E$, is one that minimizes $\omega$, i.e. such that $\omega\left(\pi^{*}\right)=\inf \{\omega(\pi) \mid \pi \in \Pi(E)\}$.

Proposition 1. Let $\pi_{1}$ and $\pi_{2}$ be two partial partitions of same support, and $\pi_{0}$ be a partial partition disjoint from $\pi_{1}$ and $\pi_{2}$. An energy $\omega$ on $\mathcal{D}(E)$ is said to be hierarchically increasing, or $h$-increasing, in $\mathcal{D}(E)$ when, $\pi_{0}, \pi_{1}, \pi_{2} \in \mathcal{D}(E), \pi_{0}$ disjoint of $\pi_{1}$ and $\pi_{2}$, we have

$$
\omega\left(\pi_{1}\right) \leq \omega\left(\pi_{2}\right) \quad \Rightarrow \quad \omega\left(\pi_{1} \sqcup \pi_{0}\right) \leq \omega\left(\pi_{2} \sqcup \pi_{0}\right) .
$$

Let $H \in \mathcal{H}$ be a finite hierarchy, and $\omega$ be an energy on $\mathcal{D}(E)$. Consider a node $S$ of $H$ with $p$ sons $T_{1} . . T_{p}$ of minimum cuts $\pi_{1}^{*}, . . \pi_{p}^{*}$. The largest cut of minimum energy of summit $S$ is either the cut

$$
\pi_{1}^{*} \sqcup \pi_{2}^{*} . . \sqcup \pi_{p}^{*},
$$

or the partition of $S$ into a unique class, if and only if $S$ is h-increasing (proof given in [14]).

Implication (2) is illustrated in Figure 3. The condition of $h$-increasingness yields a dynamic program for finding the optimum cut $\pi^{*}(H)$ in one pass i.e. each node of the hierarchy is read only once [6], [14]. 


\section{Generation of $h$-Increasing Energies}

Composition from the energies of the classes. An easy way to obtain a $h$ increasing energy on the family $\mathcal{D}(E)$ of all partial partitions of $E$ consists in taking, firstly, an arbitrary energy $\omega$ on all sets $S \in \mathcal{P}(E)$, considered as one class partial partitions $\{S\}$, and then in extending $\omega$ to all partial partitions by some law of composition. The $h$-increasingness is introduced here by the law of composition, and not by $\omega[\mathcal{P}(E)]$. The first laws which come to mind are, of course, addition, supremum, and infimum, and indeed we can state

Proposition 2. Let $E$ be a set and $\omega: \mathcal{P}(E) \rightarrow \mathbb{R}^{+}$an arbitrary energy defined on $\mathcal{P}(E)$, and let $\pi \in \mathcal{D}(E)$ be a partial partition of classes $\left\{S_{i}, 1 \leq i \leq n\right\}$. Then the three extensions of $\omega$ to the partial partitions $\mathcal{D}(E)$

$$
\omega(\pi)=\bigvee_{i} \omega\left(S_{i}\right), \quad \omega(\pi)=\bigwedge_{i} \omega\left(S_{i}\right), \quad \text { and } \omega(\pi)=\sum_{i} \omega\left(S_{i}\right),
$$

are $h$-increasing energies.

Here, $\bigvee$ and $\Lambda$ are the supremum and infimum operators respectively. The second rel.(44) encompasses that of Mumford and Shah, and that of the separable energies [6] [5. The energies composed by suprema appear in [7] [8]. A number of other laws are compatible with $h$-increasingness. One could use the product of energies, the difference sup-inf, the quadratic sum, and their combinations. Moreover, one choose an $\omega$ dependent on more than one class, on the proximity of the edges, on another hierarchy, etc..

Lattice of $h$-increasing energies. The $h$-increasing energies can be combined in several manners. For example, if $\left\{\omega_{i}, i \in I\right\}$ stands for a family of sum-generated energies, and for a family $\left\{\lambda_{i}, i \in I\right\}$ of non negative weights, then the weighed sum $\omega=\sum \lambda_{i} \omega_{i}$ turns out to be $h$-increasing, and sum-generated.

Similarly, the class $\mathcal{L}_{\vee}$ of the $\vee$-generated $h$-increasing energies $\left\{\omega_{i}, i \in I\right\}$ is closed under weighted supremum, i.e. $\omega=\vee \lambda_{i} \omega_{i} \in \mathcal{L}_{\vee}$. Moreover, $\omega_{\text {min }} \in \mathcal{L}_{\vee}$ for $\omega_{\min }(\pi)=0, \forall \pi \in \mathcal{D}(E)$. Therefore $\mathcal{L}_{\vee}$ turns out to be a lattice for the usual numerical ordering. Note that the $\vee$, paradoxically, expresses the intersection of criteria. For example take, in a color pyramid, the energy:

$$
\begin{aligned}
& \omega_{1}(S)=0 \text { if the range of the luminance in } S \text { is }<k_{1}, \quad \omega_{1}(S)=1 \text { if not, } \\
& \omega_{2}(S)=0 \text { if the range of the saturation in } S \text { is }<k_{2}, \quad \omega_{2}(S)=1 \text { if not, } \\
& \omega_{3}(S)=0 \text { if the area of } S \text { is } \geq k_{3}, \quad \omega_{3}(S)=1 \text { if not. }
\end{aligned}
$$

Then the energy $\vee \omega_{i}(S)=0$ when $S$ is not too small, and constant enough in luminance and saturation.

Energies by refinement. Up to now, we have associated an energy with each subset $X$ of $E$, and then extended it to each partial partition $\pi$ of the space by some law of composition of the classes $X$ of $\pi$. But the goal of $h$-increasingness 


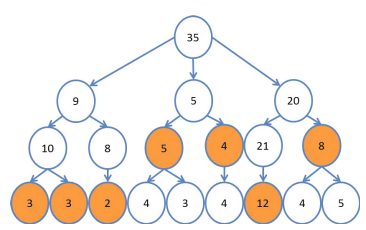

(a) Addition

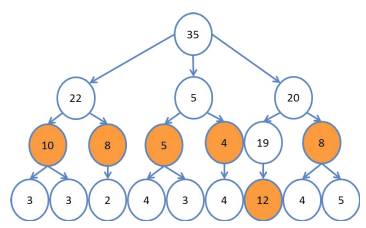

(b) Supremum

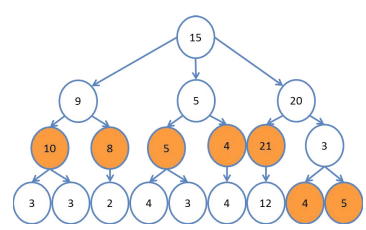

(c) Refinement

Fig. 4. Optimal Cuts by the 3 different composition of energies, (a) (SalambierGuigues) addition - The value $f(x)$ at node $x$ is compared to the sum $g(x)$ of the values of its sons. When $f(x)<g(x)$, then one keeps $f(x)$ When not, one replaces node $x$ by its sons. The optimal cut is the union of the largest kept nodes. (b) (Soille-Grazzini) supremum- The values of $f(x)$ decrease along the hierarchy. A node is maintained when $f(x) \leq k$ The optimal cut is the union of the largest kept nodes. (c) (Akçay-Aksoy) Refinement $-\omega(S) \leq \omega(S \prime)$ when 1) $S \subseteq S \prime$ 2) $f(S) \leq f(S \prime)$ The optimal cut at point $\mathrm{x}$ is the largest node which is more energetic than all its descendants Or, when none, it is the leaf that contains point $x$.

does not demand we know the energy of all partial partitions. It suffices indeed to be able to order the energies, which can be obtained from the usual ordering by refinement on partitions. Allocate an energy $\mu(S)$ to each node of a hierarchy $H$, where $\mu$ takes its values in a partially ordered set $M$.

Definition 1. The node $S$ is said to be less energetic, in terms of refinement, than $S^{\prime}$, and one writes $S \preccurlyeq S^{\prime}$, when

$$
S \preccurlyeq S^{\prime} \quad \Leftrightarrow \quad S \supseteq S^{\prime} \quad \text { and } \mu(S) \geq \mu\left(S^{\prime}\right) \quad S, S^{\prime} \in \mathcal{P}(E), \mu \in M .
$$

The partial partition $\pi$ is less energetic than $\pi^{\prime}$, and one writes $\pi \preccurlyeq \pi^{\prime}$, when

1. both $\pi$ and $\pi^{\prime}$ have the same support Supp

2. for all points $x \in S u p p$, one has $S(x) \preccurlyeq S^{\prime}(x)$.

The energy $\omega$ associated with $\pi$ is obviously $h$-increasing. Therefore there exists a minimum cut. For finding its node at point $x$, one starts from the set $\mathcal{S}(x)=$ $\left\{S_{i}(x), 1 \leq i \leq n\right\}$ of all classes containing $x$. It admits an element $S_{i_{0}}(x)$ of smallest energy, where either $i_{0}=\sup \left\{i: \mu\left(S_{i}\right) \leq \mu\left(S_{i-1}\right), 1 \leq i \leq n\right\}$, or $i_{0}=1$. Unlike the additive and the $\vee$-generated energies, this one accepts that, among several "brothers", some be minimum nodes and not others. One may find an example of energy by refinement, due to [9]. They study airborne multi bands images and take (up to a small change) $\mu(S)=-$ Area $(S) \times($ mean of all standard deviations of all bands in $S$ )

Any index of similarity can be used for $\mu$. For example, take $\mu(S)=0$ when all sons of $S$ have similar areas, or similar colour histograms, etc.. Then the supremum $\vee\left\{\mu_{r}, 1 \leq r \leq p\right\}$ of such binary texture energies is still of the same type (i.e. $\mu(S)=\vee \mu_{r}(S)=0$ when all $\mu_{r}(S)=0$ and $\mu(S)=1$ when not), and it represents the intersection of all criteria $\mu_{r}$. 


\section{Climbing Energies}

The usual energies are often given by finite sequences $\left\{\omega^{j}, 1 \leq j \leq p\right\}$ that depend on a positive index, or parameter, $j$. Therefore, the processing of hierarchy $H$ results in a sequence of $p$ optimum cuts $\pi^{j *}$, of labels $1 \leq j \leq p$. A priori, the $\pi^{j *}$ are not ordered, but if they were, i.e. if

$$
j \leq k \quad \Rightarrow \quad \pi^{j *} \leq \pi^{k *}, \quad j, k \in J
$$

then we should obtain a nice progressive simplification of the optimum cuts. For getting it, we need to combine $h$-increasingness with the supplementary axiom (17) of scale increasingness, which results in the following climbing energies.

Definition 2. We call climbing energy any family $\left\{\omega^{j}, 1 \leq j \leq p\right\}$ of energies over $\widetilde{\Pi}$ which satisfies the three following axioms, valid for $\omega^{j}, 1 \leq j \leq p$ and for all $\pi \in \Pi(S), S \in \mathcal{S}$

- i) each $\omega^{j}$ is $h$-increasing,

- ii) each $\omega^{j}$ admits a single optimum cutting,

- iii) the $\left\{\omega^{j}\right\}$ are scale increasingness, i.e. for $j \leq k$, each support $S \in \mathcal{S}$ and each partition $\pi \in \Pi(S)$, we have that

$$
j \leq k \quad \text { and } \omega^{j}(S) \leq \omega^{j}(\pi) \Rightarrow \omega^{k}(S) \leq \omega^{k}(\pi), \quad \pi \in \Pi(S), \quad S \in \mathcal{S} .
$$

Axiom i) and ii) allow us to compare the same energy at two different levels, whereas iii) compares two different energies at the same level. The relation (7) means that, as $j$ increases, the $\omega^{j}$ 's preserve the sense of energetic differences between the nodes of hierarchy $H$ and their partial partitions. In particular, all energies of the type $\omega^{j}=j \omega$ are scale increasing. The climbing energies satisfy the very nice property to order the optimum cuts with respect to the parameter $j$ :

Theorem 1. Let $\left\{\omega^{j}, 1 \leq j \leq p\right\}$ be a family of energies, and let $\pi^{j *}$ (resp. $\left.\pi^{k *}\right)$ be the optimum cut of hierarchy $H$ according to the energy $\omega^{j}$ (resp. $\left.\omega^{k}\right)$. The family $\left\{\pi^{j *}, 1 \leq j \leq p\right\}$ of the optimum cuts generates a unique hierarchy $H^{*}$ of partitions, i.e.

$$
j \leq k \quad \Rightarrow \quad \pi^{j *} \leq \pi^{k *}, \quad 1 \leq j \leq k \leq p
$$

if and only if the family $\left\{\omega^{j}\right\}$ is a climbing energy (proof given in [14]).

Such a family is climbing in two senses: for each $j$ the energy climbs pyramid $H$ up to its best cut (h-increasingness), and as $j$ varies, it generates a new pyramid to be climbed (scale-increasingness). The question of unicity of the optimal cuts is not handled here, but here in [14]. 


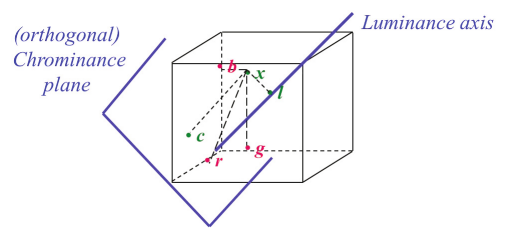

(a) Luminance-Chrominance Vectors

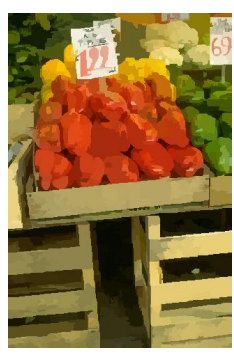

(b) Cut by Luminance

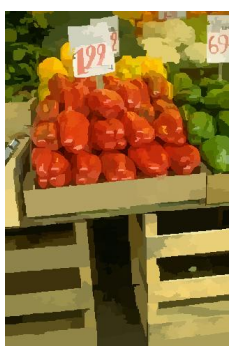

(c) Cut by Chromi-

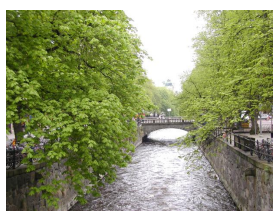

(d) Input Image

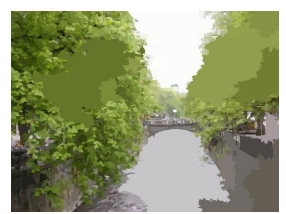

(e) $\sigma_{\text {Chrom }}^{2},(\mathrm{f})$

, (f) $\sigma_{\text {Chrom }}^{2}$,

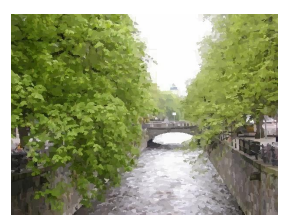

$\lambda=100, K=10^{12}$

$\lambda=100, K=10^{14}$

Fig. 5. In the RGB space, a colour vector $\vec{x}(r, g, b)$ can be decomposed in its two orthogonal projections on the grey axis, namely $\vec{l}$ of components $(l / 3, l / 3, l / 3)$, and on the chromatic plane orthogonal to the grey axis at the origin, namely $\vec{c}$ of components $(3 / \sqrt{2})(2 r-g-b, 2 g-b-r, 2 b-r-g)$. We have $\vec{x}=\vec{l}+\vec{c}$. (d) (e) and (f) Optimal cut that enhances texture by parameters: $\sigma_{\text {Lum }}^{2}, \sigma_{\text {Chrom }}^{2}$ refers to whether the Energy function was composed on variance of the partial partitions Luminance or Chrominance. $\lambda$ the scale parameter, $K$ constant that normalizes the variance of areas of set of children with respect to global energy of the children belonging a given parent

\section{Examples}

In all cases below, the energies depend on a scalar parameter $k$ such that the families $\left\{\omega^{k}\right\}$ are climbing.

Supremum composed binary energies . The simplest energies are the binary ones, which take values 1 and 0 only. We firstly observe that the relation $\pi \sqsubseteq \pi_{1}$, where $\pi_{1}=\pi \sqcup \pi^{\prime}$ is made of the classes of $\pi$ plus other ones, is an ordering. A binary energy $\omega$ such that for all $\pi, \pi_{0}, \pi_{1}, \pi_{2} \in \mathcal{D}(E)$

$$
\begin{gathered}
\omega \text { is } \sqsubseteq \text {-increasing, i.e. } \omega(\pi)=1 \Rightarrow \omega\left(\pi \sqcup \pi_{0}\right)=1 \\
\omega\left(\pi_{1}\right)=\omega\left(\pi_{2}\right)=0 \Rightarrow \omega\left(\pi_{1} \sqcup \pi_{0}\right)=\omega\left(\pi_{2} \sqcup \pi_{0}\right),
\end{gathered}
$$

is obviously $h$-increasing, and conversely. The Soille-Grazzini minimization provides an example of this type. A numerical function $f$ is now associated with hierarchy $H$. Consider the range of variation $\delta(S)=\max \{f(x), x \in S\}-$ $\min \{f(x), x \in S\}$ of $f$ inside set $S$, and the $h$-increasing binary energy $\omega^{k}(\langle S\rangle)=$ 0 when $\delta(S) \leq k$, and $\omega^{k}(\langle S\rangle)=1$ when not. Compose $\omega$ according the law of 
the supremum, i.e. $\pi=\sqcup\left\langle S_{i}\right\rangle \Rightarrow \omega^{k}(\pi)=\bigvee_{i} \omega^{k}\left(\left\langle S_{i}\right\rangle\right)$. Then the class of the optimum cut at point $x \in E$ is the larger class of $H$ whose range of variation is $\leq j$. When the energy $\omega^{k}$ of a father equals that of its sons, one keeps the father when $\omega^{k}=0$, and the sons when not.

Additive energies under constraint. The example of additive energy that we now develop is a variant of the creation of thumbnails by $\mathrm{Ph}$. Salembier and L. Garrido [5]. We aim to generate "the best" simplified version of a colour image $f$, of constrained by compression rate. A hierarchy $H$ has been obtained by previous segmentations of the luminance $l=(r+g+b) / 3$ based on 1$]$. In each class $S$ of $H$, the simplification consists in replacing the function $f$ by its colour mean (mean over all 3 channels) $\mu(S)=\frac{\Sigma_{x \in S} x}{\operatorname{card}(S)}$. The data fidelity term(we refer in short as $\left.\omega_{\mu}(\pi)\right)$ is given by $L_{2}$ norm by the first functional in 9 while the constraint function (second functional) i.e. The coding cost(we refer in short as $\omega_{\partial}(\pi)$ ) for a frontier element is $\simeq 2$, which is given, for the whole $S$, with 24 bits assigned to code each color value.

$$
\omega_{\text {lum }}(S)=\sum_{x \in S}\|l(x)-m(S)\|^{2}+\lambda(24+|\partial S|),
$$

Here we separate the colour image into 2 components the luminance vector which gives the gray scale, and its complementary chrominance plane as shown in $5(\mathrm{a})$ The principle idea here is to show that the non negative energy described per partition and the actual hierarchies of partitions are well separated entities in this framework, and thus multiple constraint functions to optimize over a hierarchy of partitions generated from a different function over the image space.

$$
\omega_{\text {chrom }}(S)=\sum_{x \in S}\|c(x)-m(S)\|^{2}+\lambda(24+|\partial S|)+\sum_{S \prime \in \text { siblings }(S)} \frac{K}{\sigma^{2}(\text { Area }(S \prime))},
$$

Thus here in (10) we observe that the class variance is now calculated over the chrominance function of the image, which simplifies the image while keeping partitions which minimize the variance of the chrominance vector $c$. According to Lagrange formalism, the total energy of class $S$ is as shown. Classically one reaches the minimum under constraint $\omega(S)$ by means of a system of partial derivatives. Now remarkably our approach replaces the computation of derivatives by a climbing. Indeed we can access the energy of a cut $\pi$ by summing up that of its classes, which leads to $\omega(\pi)=\omega_{\mu}(\pi)+\lambda^{j} \omega_{\partial}(\pi)$. The cost $\omega_{\partial}(\pi)$ decreases as $\lambda^{j}$ increases, therefore we can climb the pyramid of the best cuts and stop (thus optimal $\lambda$ ) when the constraint is satisfied.

Intuitively, texture features are formulated into this multi-scale framework where the optimal scale parameter combines the effect of chrominance and structure of texture into one global energy function, thus showing the flexibility of the framework. The third term in (10) decreases when any child in the hierarchy whose siblings have low variance of component areas with respect to each other. This is done also with the constraint that the variance of the chrominance vector 
is reduced over the partitions of pyramid produced from the luminance vector $l$. The lattice of h-increasing energies extends this concept to energies whose optimal cuts can be combined or recomposed again using the supremum of the partitions, as explained earlier in the section - "lattice if $h$-increasing energies".

\section{Conclusion}

The primary contributions of this theoretical paper were :

1. Defining an novel non negative global climbing energy that helps perform an optimization over the hierarchy of segmentations, that generate optimal segmentations by the classical Mumford shah functional, and compiling other energies used to combine partitions from hierarchies, in the literature.

2. Obtaining the conditions for a general class of energies to be $h$-increasing.

3. Demonstrating how to formulate multiple constraint functions over the image space and obtained different optimal segmentations. An example with colour image segmentation and Texture enhancement are shown.

The results in this paper are thus not necessarily to be restricted to the point of view of global constraints but belong to the theoretical results of optimization over scale increasing pyramids of partitions.

\section{References}

1. Cousty, J., Najman, L.: Incremental Algorithm for Hierarchical Minimum Spanning Forests and Saliency of Watershed Cuts. In: Soille, P., Pesaresi, M., Ouzounis, G.K. (eds.) ISMM 2011. LNCS, vol. 6671, pp. 272-283. Springer, Heidelberg (2011)

2. Arbelaez, P., Maire, M., Fowlkes, C., Malik, J.: Contour detection and hierarchical image segmentation. IEEE Trans. Pattern Anal. Mach. Intell. 33, 898-916 (2011)

3. Russell, B.C., Freeman, W.T., Efros, A.A., Sivic, J., Zisserman, A.: Using multiple segmentations to discover objects and their extent in image collections. In: Proceedings of the 2006 IEEE CVPR, vol. 2, pp. 1605-1614 (2006)

4. Cardelino, J., Caselles, V., Bertalmío, M., Randall, G.: A contrario hierarchical image segmentation. In: ICIP, pp. 4041-4044 (2009)

5. Salembier, P., Garrido, L.: Binary partition tree as an efficient representation for image processing, segmentation, and information retrieval. IEEE Transactions on Image Processing 9, 561-576 (2000)

6. Guigues, L., Cocquerez, J.P., Men, H.L.: Scale-sets image analysis. International Journal of Computer Vision 68, 289-317 (2006)

7. Soille, P.: Constrained connectivity for hierarchical image partitioning and simplification. IEEE Transactions on Pattern Analysis and Machine Intelligence 30, 1132-1145 (2008)

8. Zanoguera, M.F., Marcotegui, B., Meyer, F.: A toolbox for interactive segmentation based on nested partitions. In: ICIP (1), pp. 21-25 (1999)

9. Akcay, H.G., Aksoy, S.: Automatic detection of geospatial objects using multiple hierarchical segmentations. IEEE T. Geoscience and Remote Sensing 46, 2097-2111 (2008) 
10. Serra, J.: Hierarchies and Optima. In: Debled-Rennesson, I., Domenjoud, E., Kerautret, B., Even, P. (eds.) DGCI 2011. LNCS, vol. 6607, pp. 35-46. Springer, Heidelberg (2011)

11. Lucchi, A., Li, Y., Bosch, X.B., Smith, K., Fua, P.: Are spatial and global constraints really necessary for segmentation? In: ICCV, pp. 9-16 (2011)

12. Ronse, C.: Partial partitions, partial connections and connective segmentation. J. Math. Imaging Vis. 32, 97-125 (2008)

13. Najman, L., Schmitt, M.: Geodesic saliency of watershed contours and hierarchical segmentation. IEEE Trans. Pattern Anal. Mach. Intell. 18, 1163-1173 (1996)

14. Serra, J., Kiran, B.R.: Climbing on pyramids. CoRR abs/1204.5383 (2012) 